\title{
Evaluation of Food Quality of Released Barley Varieties Grown in Oromia, Ethiopia
}

\author{
Megersa Daba*, Abiyot Lelisa \\ Oromia Agricultural Research Institute, Addis Ababa, Ethiopia \\ Email address: \\ megersa2@gmail.com (M. Daba) \\ ${ }^{*}$ Corresponding author
}

\section{To cite this article:}

Megersa Daba, Abiyot Lelisa. Evaluation of Food Quality of Released Barley Varieties Grown in Oromia, Ethiopia. International Journal of Science, Technology and Society. Vol. 9, No. 3, 2021, pp. 127-134. doi: 10.11648/j.ijsts.20211003.13

Received: March 8, 2021; Accepted: May 19, 2021; Published: June 8, 2021

\begin{abstract}
This study was conducted to characterize and evaluate food quality of released food barley varieties through physical, chemical and sensory evaluation. Fifteen released food barley varieties were collected from different research centers. Physicochemical qualities of these varieties were analyzed with three replications. Sensory evaluation was also performed by using hedonic scale method. Hectoliter weight, thousand kernel weight, moisture, protein, sodium, potassium, iron, zinc, calcium and magnesium contents were determined in the range of $55.57-66.67$ grams, $33.50-58.50$ grams, $7.46-11.43 \%$, 9.44 - 16.80\%, 79.23 - 316.54 ppm, 3993 - 6040 ppm, 11.49 - 64.32 ppm, 31.07 - 55.73 ppm, $305.42-716.91$ ppm and $811.50-1731.10 \mathrm{ppm}$ respectively. Overall acceptability of porridge prepared from barley varieties were disliked slightly to liked moderately while liked slightly to liked moderately for Injera. There were significant $(\mathrm{P}<0.05)$ variation in physical, chemical and organoleptic properties due to test barley varieties variation. Aruso variety was the highest in mean value of thousand kernel weight and hectoliter weight. Walker variety had the highest protein mean score. The study revealed that Robera, Abdane, Bentu, Harbu, Golden Eye and Walker varieties had the highest value of calcium, magnesium, potassium, sodium, iron and zinc respectively. Walker variety porridge was disliked slightly and Bentu variety was mostly liked moderately but not significantly different among Aquila, Gobe, HB 1966 and Robera varieties. Abdane variety injera was liked slightly and Biftu variety was mostly liked moderately but not significantly different among nine barley varieties. Therefore Aruso, Biftu, Bentu and Robera varieties were preferred for physical, chemical and sensory quality attributes.
\end{abstract}

Keywords: Food Barley, Variety, Physical, Chemical, Sensory, Injera, Porridge

\section{Introduction}

Barley (Hordeum vulgare L.) is used for animal's feed and human consumption. It is estimated that about $85 \%$ of the world's barley production is destined for feeding animals, while the rest is used for malt production, seed production and food consumption but also for production of starch either for food use or for the chemical industry [1]. Barley is used as an important food crop in daily diets in Morocco, India, China and Ethiopia [2]. Barley is used as main crop for food and beverage preparation in Ethiopia. It can be used as main dishes like Injera, bread (kita) and porridge in addition to ceremonial and side dishes like local beverage (farso in Afan Oromo), roasted whole grain, Besso, Chuko, Kinche, etc [3]. It's considered as health food and mostly used for infant food preparation. Because of these various interests, barley is mainly produced in Oromia regional state. Of the top 25 barley producing districts in the country, 18 are found in Oromia [4]. In $2017 / 2018$ cropping season, barley was produced on about 951,993.15 hectares of land from which 20,529,963.72 quintals of yield are obtained [5]. According to Ethiopian Ministry of Agriculture report, there are about 46 released food barley varieties until 2018 [6]. Ten varieties (Walker, Golden Eye, Aquila, Robera, Abdane, Guta, Biftu, Dinsho, Harbu, and Adoshe) were released by Oromia Agricultural Research Institute's research centers.

The environmental factors, such as rainfall, temperature, soil conditions, fertilizer and genetic factors, can contribute to variations in the chemical composition and physical characteristics of cereal grains [7\&8]. Thus characterization of variations in the nutritional value of cereal grains that 
result from such factors may help to define appropriate breeding objectives for improving the value of cereal grains for nutrition [8]. The exact physicochemical composition of barley may vary depending on the variety and the environmental conditions during production. Accordingly, different researchers have evaluated internationally and some nationally released varieties barley for their nutritional composition [9 \& 10]. Whole barley grain consisted of about $65-68 \%$ starch, $10-17 \%$ protein, $4-9 \%$ s-glucan, $2-3 \%$ free lipids and $1.5-2.5 \%$ minerals [11].

It is important to investigate the nutritional value of barley in a given to geographic location because their nutritional value may depend on the variety, fertilization and environmental conditions. Considerable number of food barley varieties were verified and released from different Agricultural Research Centers of IQQO. However, some of nutritional compositions of these varieties were not evaluated and profiled as research information as well as consumers preferences were not reported. Therefore the objective of this study was to evaluate the physicochemical compositions and consumers' preferences of released and mostly produced barley varieties in the Oromia region, Ethiopia.

\section{Objectives}

1) To evaluate physical and chemical quality of food barley varieties found in Oromia, Ethiopia,

2) To evaluate processed food quality of barley found in Oromia, Ethiopia

\section{Materials and Methods}

\subsection{Sample Collection and Study Site}

Fifteen released food barley varieties were collected from Fedis Agricultural Research Center (FARC) and Sinana Agricultural Research Center (SARC) during $2017 / 18$ cropping season as listed on Table 1 . Finally, only undamaged food barley grain was chosen and stored under room temperate until analysis. Laboratory analysis and barley food products sensory evaluation activities were undertaken at Food Science Laboratory of Oromia Agricultural Research Institute (IQQO) and Dodola district. Four farmers' research groups (FRG) having each fifteen members were established at Dodola district (Denaba and Kecama Core kebeles). Food barley Injera and porridge prepared from different barley varieties were evaluated by IQQO's staff and farmers from Dodola district found in West Arsi zone.

\subsection{Sample Preparations for Analysis}

Food barley varieties were sorted, cleaned, decorticated by using mortar and pestle and sun dried (as shown on figure 1), milled, sieved and stored at room temperature until chemical and sensory analysis carried out.
Table 1. List Barley varieties, Breeder and Released Year.

\begin{tabular}{llll}
\hline $\begin{array}{l}\text { S. } \\
\text { No }\end{array}$ & $\begin{array}{l}\text { Variety } \\
\text { name }\end{array}$ & Breeder/Maintainer & $\begin{array}{l}\text { Year of } \\
\text { Released }\end{array}$ \\
\hline 1 & Abdane & SARC/IQQO & 2011 \\
2 & Aquila & FARC(IQQO)/MORRELL & 2012 \\
3 & Aruso & SARC/IQQO & Local \\
4 & Biftu & SARC/IQQO & 2005 \\
5 & Bentu & KARC/IQQO & 2006 \\
6 & Dafo & SARC/IQQO & 2005 \\
7 & Dinsho & SARC/IQQO & 2009 \\
8 & Gobe & KARC/IQQO & 2012 \\
9 & Golden Eye & FARC(IQQO)/MORRELL & 2012 \\
10 & Harbu & SARC/IQQO & 2004 \\
11 & HB 1965 & HARC & 2017 \\
12 & HB 1966 & HARC & 2017 \\
13 & HB 1307 & HARC/EIAR & 2006 \\
14 & Robera & SARC/IQQO & 2016 \\
15 & Walker & FARC(IQQO)/MORRELL & 2012 \\
\hline
\end{tabular}

Where, $\mathrm{KARC}=$ Kulumsa Agricultural Research Center, HARC $=$ Holeta Agricultural Research Center, EIAR= Ethiopian Institute of Agricultural Research
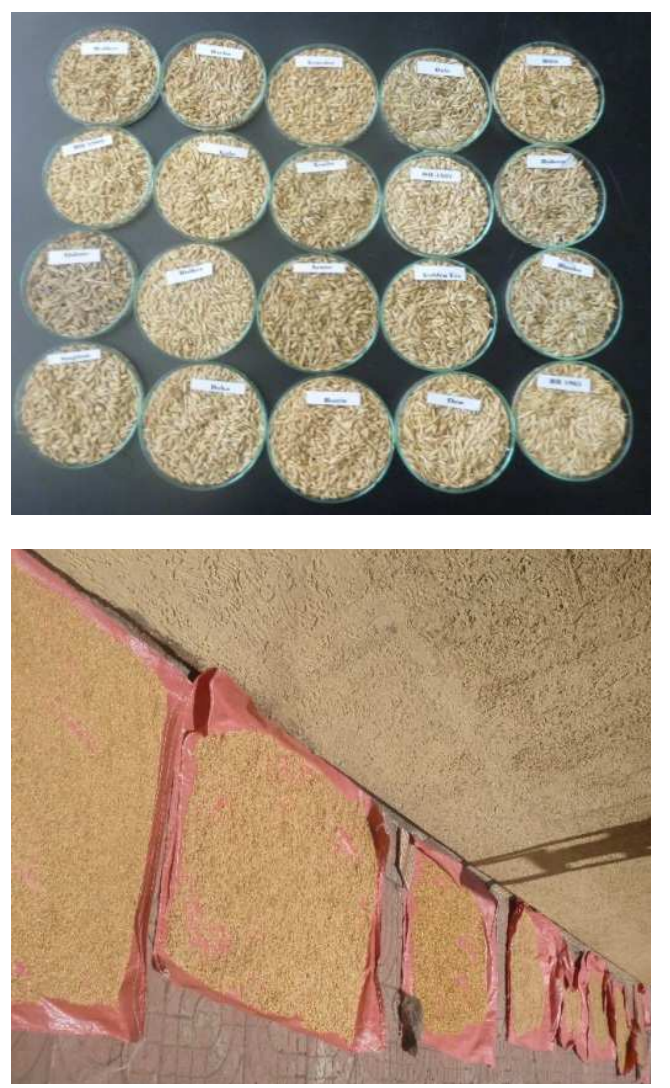

Figure 1. Barley Varieties decorticated and during sun drying.

\subsection{Physicochemical Analysis}

\subsubsection{Thousand Seed Weight}

Thousand barley kernel counted by automatically seed counter and weighed by sensitive balance $(0.001 \mathrm{~g})$ and thousand seed weight was reported in grams. The weight of each test was repeated with three replicates [12].

\subsubsection{Proximate Analysis}

Proximate Composition: Moisture, protein and fat content of the grain samples and proximate composition of bread 
blends were determined by using the AOAC, 2000 methods [13]. Total carbohydrate is calculated by difference. Energy was calculated using Artwater factor: Fat x $9+$ Carbohydrate x $4+$ Protein x 4 (kcal).

\subsubsection{Minerals}

Iron, zinc and calcium content were analyzed by using AOAC Official Method 975.03 [14]. All determinations were done in triplicate.

\subsubsection{Injera Preparation from Barley Varieties}

Injera was prepared as per the procedure of Bultosa et al., [15] and Fitsum et al., [16]. Equal amount of flour and water were used for the preparation of dough and fermentation of the dough after adding a starter culture (a fermented dough from previous batch) with $1: 1.6 \mathrm{w} / \mathrm{v}$ and fermenting at room temperature for $24-72 \mathrm{~h}$. After fermentation, $10 \%$ of the sediment was mixed with water (1:3) and cooked for 2-3 minutes with the objective of gelatinization (cooking) primarily to bring about the cohesiveness of dough and secondly to get ride of the easily fermentable carbohydrate from Injera. Then, gelatinized batter (Absit) were cooled to room temperature and added back to the fermenting dough. After fermentation for $0.5-1 \mathrm{~h}$, bubbles were formed, indicating the end point. Additional water was added to fermented dough to bring to correct batter consistency. About $500 \mathrm{~g}$ of fermented batter was poured in a circular manner on a hot clay griddle, covered, and baked for 3-4 minutes.

\subsection{Consumer Preference Test on Processed Food}

Before conducting sensory evaluation; orientation was given for panelists with practical demonstration. Sixty three (36=male and $27=$ female $)$ and sixty eight $(39=$ male and $29=$ female) consumers including researchers, farmers, and nutrition and plant science experts were purposely selected to determine the acceptability of barley porridge and Injera displayed on figure 2 and 3 respectively. Acceptability/preference was undertaken using nine point Hedonic scales; where $1=$ dislike extremely, $2=$ dislike very much, 3. Dislike moderately, 4. Dislike slightly, 5=neither like nor dislike, $6=$ like slightly, 7. Like moderately, 8. Like very much and $9=$ like extremely.

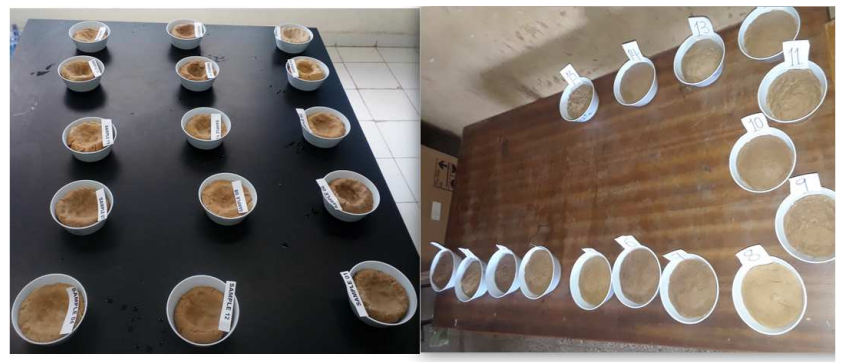

Figure 2. Barley varieties porridge displayed for sensory evaluation.

\subsection{Data Management and Statistical Analysis}

Means and standard deviations were calculated for physicchemicals and acceptability of the sensory attributes. All quantitative and qualitative data were analyzed using statistical analysis software known as SAS version 9.00 to analysis physic-chemicals and sensory qualities.

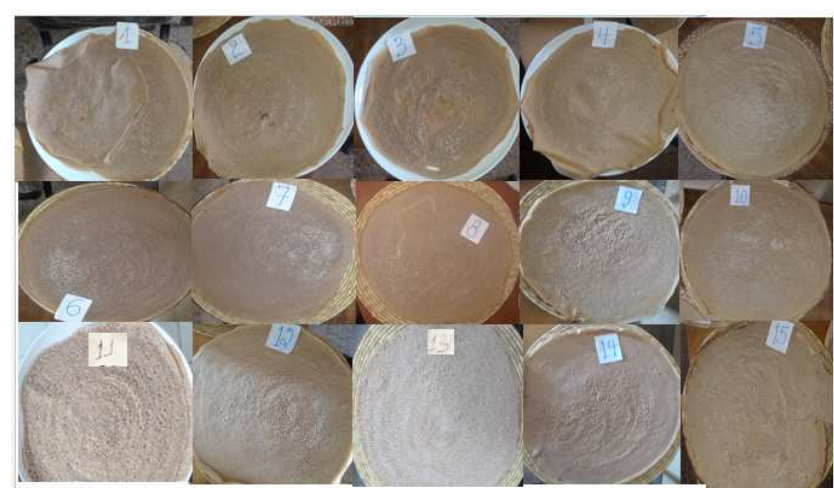

Figure 3. Barley varieties Injera displayed for consumers preference.

\section{Results and Discussions}

\subsection{Some Physical and Chemical Qualities of Barley Varieties Grain}

Hectoliter weight, thousand kernel weights, moisture, protein, and some minerals content were determined in selected barley varieties were shown on Table 2. All of determined physico-chemicals of food qualities were significant $(\mathrm{p}<0.05)$ among barley varieties. The least hectoliter weigh, thousand kernel weight and moisture were determined from Walker $(62.48 \mathrm{~g})$, Bentu (34.71g) and Bentu $(7.90 \%)$ respectively, while the maximum amount hectoliter weigh, thousand kernel weight and moisture was obtained from Aruso Variety with value of 74.01 grams, 66.20 grams and $11.43 \%$ respectively. Thousand-grain and hectoliter weights, which can be used to determine the potential flour yield in wheat grain, are accepted as the main quality factors by the milling industry [17]. Therefore, Aruso variety had flour advantages than others.

The protein composition barley varieties determined with the least from Aruso variety (10.72\%) and the highest from Walker variety $(18.72 \%)$. The level of protein in barley is highly variable, ranging from 7 to $25 \%$ according to a large USDA study involving over 10,000 genotypes [18]. The difference is due to the varieties growth conditions, particularly the rate and timing of nitrogen fertilization [19] and also barley protein content is highly dependent on the cultivar [20]. The minerals content of minerals in barley varieties ranged from $87.14-366,4248$ - 8778.18, 34.00 - 78.48, 428.77 - 822.54, and $928.05-$ $2126.23 \mathrm{ppm}$ for sodium, potassium, iron, zinc, calcium and magnesium respectively. The exact composition of barley will vary depending on the variety chosen and the environmental conditions during growth [9]. The whole barley grain consists of about 65-68\% starch, $10-17 \%$ protein, $4-9 \% \beta$-glucans, $2-3 \%$ free lipids and $1.5-2.5 \%$ minerals [21 \& 22]. 
Table 2. Physical and chemical qualities of grain barley varieties selected Oromia Agricultural Research Institute research centers.

\begin{tabular}{|c|c|c|c|c|c|c|c|c|c|c|}
\hline \multirow{2}{*}{$\begin{array}{l}\text { Barley } \\
\text { varieties }\end{array}$} & \multicolumn{10}{|c|}{ Physico-chemicals qualities of Barley varieties at dry basis } \\
\hline & $\begin{array}{l}\text { Hectoliter weight } \\
\text { (grams) }\end{array}$ & $\begin{array}{l}\text { Thousand kernel } \\
\text { weight (grams) }\end{array}$ & $\begin{array}{l}\text { Moisture } \\
\text { (\%) }\end{array}$ & Protein (\%) & Na ppm & K ppm & Fe ppm & Zn ppm & Ca ppm & Mg ppm \\
\hline Abdane & $70.22 \pm 0.20^{\mathrm{de}}$ & $49.95 \pm 1.01^{\mathrm{d}}$ & $9.57 \pm 0.05^{\mathrm{d}}$ & $16.25 \pm 1.91^{\text {cd }}$ & $153.84^{\text {efg }}$ & $7580.18^{c}$ & $32.39^{\mathrm{g}}$ & $44.78^{\text {cd }}$ & $730.47^{\mathrm{ab}}$ & $2126.23^{\mathrm{a}}$ \\
\hline Aquila & $62.68 \pm 0.75^{\mathrm{h}}$ & $45.89 \pm 4.97^{\mathrm{e}}$ & $10.63 \pm 0.12^{\mathrm{bc}}$ & $16.55 \pm 0.02^{\mathrm{c}}$ & $200.29^{\mathrm{d}}$ & $7584.70^{\mathrm{c}}$ & $38.34^{\mathrm{f}}$ & $54.55^{\mathrm{cb}}$ & $467.97^{\mathrm{f}}$ & $1533.54^{\mathrm{bc}}$ \\
\hline Aruso & $74.01 \pm 069^{\mathrm{a}}$ & $66.2 \pm 0.48^{\mathrm{a}}$ & $11.43 \pm 0.06^{\mathrm{a}}$ & $10.72 \pm 0.1^{\mathrm{g}}$ & $192.48^{\mathrm{de}}$ & $6326.87^{\mathrm{ef}}$ & $40.41^{\text {ef }}$ & $45.09^{\mathrm{cd}}$ & $696.73^{\mathrm{abc}}$ & $1466.49^{\mathrm{bc}}$ \\
\hline Bentu & $71.39 \pm 1.03^{\mathrm{bcd}}$ & $34.71 \pm 0.34^{\mathrm{h}}$ & $7.90 \pm 0.06^{\mathrm{h}}$ & $15.45 \pm 0.11^{\mathrm{def}}$ & $201.83^{\mathrm{d}}$ & $8778.15^{\mathrm{a}}$ & $18.18^{\mathrm{h}}$ & $63.06^{\mathrm{ab}}$ & $589.83^{\text {bcdef }}$ & $1470.08^{\mathrm{bc}}$ \\
\hline Biftu & $61.82 \pm 0.07^{\mathrm{h}}$ & $39.83 \pm 0.20$ & $9.11 \pm 0.11^{\mathrm{e}}$ & $17.59 \pm 0.07^{\mathrm{b}}$ & $272.75^{\mathrm{c}}$ & $8360.97^{b}$ & $41.86^{\mathrm{e}}$ & $61.79^{\mathrm{b}}$ & $672.12^{\mathrm{abcd}}$ & $1488.61^{\mathrm{bc}}$ \\
\hline Dafo & $69.51 \pm 0.77^{\mathrm{ef}}$ & $59.48 \pm 2.24^{\mathrm{c}}$ & $11.37 \pm 0.06^{\mathrm{a}}$ & $15.46 \pm 00^{\operatorname{def}}$ & $145.83^{\mathrm{fg}}$ & $4331.42^{\mathrm{h}}$ & $42.57^{\mathrm{e}}$ & $34.00^{\mathrm{d}}$ & $644.51^{\text {bcde }}$ & $1267.53^{\mathrm{cd}}$ \\
\hline Dinsho & $68.52 \pm 0.23^{\mathrm{f}}$ & $57.85 \pm 2.06^{\mathrm{c}}$ & $11.43 \pm 0.06^{\mathrm{a}}$ & $15.81 \pm 0.19^{\text {cde }}$ & $154.38^{\mathrm{efg}}$ & $8289.95^{\mathrm{b}}$ & $59.02^{\mathrm{b}}$ & $51.28^{\mathrm{cb}}$ & $590.46^{\text {bcdef }}$ & $1255.17^{\text {cde }}$ \\
\hline Gobe & $72.45 \pm 0.25^{\mathrm{abc}}$ & $43.13 \pm 0.39^{\mathrm{f}}$ & $8.16 \pm 0.05^{\mathrm{g}}$ & $15.83 \pm 0.13^{\text {cde }}$ & $123.77^{\text {gh }}$ & $6060.29^{\text {ef }}$ & $49.72^{\mathrm{c}}$ & $58.42^{\mathrm{bc}}$ & $545.93^{\text {cdef }}$ & $1212.99^{\text {cdef }}$ \\
\hline Golden E & $68.37 \pm 3.15^{\mathrm{f}}$ & $46.88 \pm 0.05^{\mathrm{e}}$ & $10.82 \pm 0.36^{\mathrm{b}}$ & $17.97 \pm 0.19^{\mathrm{ab}}$ & $126.26^{\mathrm{gh}}$ & $4768.81^{g}$ & $76.81^{\mathrm{a}}$ & $42.71^{\mathrm{cd}}$ & $714.02^{\mathrm{abc}}$ & $1138.15^{\text {def }}$ \\
\hline Harbu & $69.48 \pm 1.77^{\mathrm{ef}}$ & $46.77 \pm 0.82^{\mathrm{e}}$ & $8.94 \pm 0.02^{\mathrm{ef}}$ & $16.53 \pm 0.26^{\mathrm{c}}$ & $366.10^{\mathrm{a}}$ & $6028.61^{\mathrm{f}}$ & $32.68^{\mathrm{g}}$ & $45.06^{\mathrm{cd}}$ & $428.77^{\mathrm{f}}$ & $928.05^{\mathrm{f}}$ \\
\hline HB 1307 & $72.98 \pm 0.1^{\mathrm{ab}}$ & $62.82 \pm 0.02^{\mathrm{b}}$ & $10.47 \pm 0.06^{\mathrm{c}}$ & $11.24 \pm 0.06^{\mathrm{g}}$ & $116.51^{\text {gh }}$ & $4248.11^{\mathrm{h}}$ & $57.36^{\mathrm{b}}$ & $43.92^{\mathrm{cd}}$ & $728.60^{\mathrm{ab}}$ & $1345.83^{\mathrm{ef}}$ \\
\hline HB 1965 & $64.41 \pm 0.01^{\mathrm{g}}$ & $36.87 \pm 0.08^{h}$ & $7.46 \pm 0.05^{\mathrm{i}}$ & $15.34 \pm 0.18^{\text {ef }}$ & $87.14^{\mathrm{h}}$ & $5942.66^{\mathrm{f}}$ & $46.07^{d}$ & $44.44^{\text {cd }}$ & $526.05^{\text {def }}$ & $1045.38^{\text {def }}$ \\
\hline HB 1966 & $65.66 \pm 0.22^{\mathrm{g}}$ & $36.71 \pm 0.21^{\mathrm{h}}$ & $8.19 \pm 0.06^{\mathrm{g}}$ & $15.07 \pm 0.12^{\text {ef }}$ & $173.46^{\text {def }}$ & $6429.52^{\mathrm{e}}$ & $41.34^{\mathrm{ef}}$ & $46.66^{\mathrm{bcd}}$ & $499.53^{\text {ef }}$ & $981.80^{\operatorname{def}}$ \\
\hline Robera & $70.81 \pm 0.22^{\text {cde }}$ & $49.33 \pm 0.21^{\mathrm{d}}$ & $8.89 \pm 0.08^{f}$ & $14.69 \pm 0.14^{\mathrm{f}}$ & $321.20^{\mathrm{b}}$ & $6915.11^{\mathrm{d}}$ & $49.19^{\text {cd }}$ & $56.54^{\mathrm{cb}}$ & $822.54^{\mathrm{a}}$ & $1691.87^{\mathrm{b}}$ \\
\hline Walker & $62.48 \pm 0.56^{\mathrm{h}}$ & $45.84 \pm 0.64^{e}$ & $10.83 \pm 0.12^{\mathrm{b}}$ & $18.72 \pm 0.02^{\mathrm{a}}$ & $192.22^{\mathrm{de}}$ & $7632.89^{c}$ & $57.85^{\mathrm{b}}$ & $78.48^{\mathrm{a}}$ & $695.12^{\mathrm{abc}}$ & $1645.17^{\mathrm{b}}$ \\
\hline Mean & 68.32 & 48.15 & 9.68 & 15.55 & 188.54 & 6618.55 & 45.59 & 51.38 & 623.51 & 1345.83 \\
\hline $\operatorname{LSD}(p<0.05)$ & 1.64 & 2.99 & 1.24 & 3.35 & 41.50 & 378.02 & 3.47 & 16.67 & 168.91 & 324.58 \\
\hline $\mathrm{CV}$ & 1.44 & 2.41 & 0.20 & 0.87 & 13.16 & 3.50 & 4.55 & 19.40 & 16.20 & 14.42 \\
\hline
\end{tabular}

Note: In each column means followed by different letters (a, b, c, d, e, etc.) are significantly different at $\mathrm{p}<0.05$

\subsection{Proximate and Energy Composition of Barley Varieties' Porridge}

Proximate (moisture, ash, protein, crude fat, crude fiber, carbohydrate) and energy content of barley varieties' porridge are presented in Table 3. Both proximate and energy composition were strongly significant $(\mathrm{P}<0.0001)$ among barley varieties' porridge. The mean moisture, ash, protein, crude fat, crude fiber, carbohydrate and energy composition of porridge were $8.21 \%, 3.17 \%, 13.90 \%, 3.03 \%, 0.93 \%$, $70.76, \%$ and $346.99 \mathrm{Kcal}$ respectively. The maximum moisture and ash content were obtained from Walker and Robera variety with value of $8.96 \%$ and $5.81 \%$ respectively.
But the least moisture and ash content was obtained from Bentu variety with value $7.32 \%$ and $1.94 \%$ respectively. The protein content of porridge ranged from $8.58 \%$ (from Dinsho) to $16.64 \%$ (from Golden). The protein composition was not significant among Golden Eye, Robera, HB 1307, Walker and Dafo varieties. The maximum crude fat and crude fiber were obtained from Dafo and HB 1966 respectively. Carbohydrate (CHO), energy, crude fat and fiber value were obtained with range of $68.09-75.11 \%, 335.24-356.75 \mathrm{Kcal}$, $1.99-4.31 \%$ and $0.50-1.21 \%$ respectively. Bentu variety was superior by energy with the value of $356.75 \mathrm{Kcal}$ but not significant among Aquila, Gobe and HB 1665 varieties.

Table 3. Proximate and Energy Content Barley Varieties' Porridge Collected from Research Centers.

\begin{tabular}{|c|c|c|c|c|c|c|c|c|}
\hline S.N & & Moisture (\%) & Ash (\%) & Protein (\%) & Crude Fiber (\%) & Crude fat (\%) & CHO (\%) & Energy(Kcal) \\
\hline 1 & Abdane & $8.13 \pm 0.68^{\text {fbdec }}$ & $4.09 \pm 0.19^{b}$ & $13.81 \pm 0.41^{\text {ef }}$ & $3.31 \pm 0.45^{\text {bcd }}$ & $1.16 \pm 0.19^{\text {ba }}$ & $69.50 \pm 1.49^{\text {fe }}$ & $343.69 \pm 4.00^{\mathrm{de}}$ \\
\hline 2 & Aquila & $8.19 \pm 0.53^{\text {bdec }}$ & $2.83 \pm 0.15^{\mathrm{dce}}$ & $15.00 \pm 0.08^{\mathrm{bdc}}$ & $2.76 \pm 0.18^{\mathrm{fecd}}$ & $0.89 \pm 0.11^{\mathrm{fde}}$ & $70.33 \pm 0.19^{\text {de }}$ & $349.30 \pm 1.54^{\text {ba }}$ \\
\hline 3 & Aruso & $8.56 \pm 0.17^{\mathrm{bac}}$ & $2.97 \pm 0.04^{\mathrm{dce}}$ & $12.94 \pm 0.51^{\mathrm{gf}}$ & $3.36 \pm 0.59^{\mathrm{bc}}$ & $0.66 \pm 0.16^{\mathrm{fg}}$ & $71.62 \pm 0.72^{\mathrm{dc}}$ & $344.17 \pm 1.49^{\mathrm{de}}$ \\
\hline 4 & Bentu & $7.32 \pm 0.26^{\mathrm{g}}$ & $1.94 \pm 0.37^{\mathrm{f}}$ & $13.60 \pm 0.07^{\text {egf }}$ & $2.71 \pm 0.12^{\text {fed }}$ & $0.93 \pm 0.03^{\mathrm{dec}}$ & $73.50 \pm 0.14^{\text {ba }}$ & $356.75 \pm 0.60^{\mathrm{a}}$ \\
\hline 5 & Biftu & $8.77 \pm 0.19^{\text {ba }}$ & $3.03 \pm 0.02^{\mathrm{dc}}$ & $12.66 \pm 1.75^{\mathrm{g}}$ & $3.29 \pm 0.61^{\mathrm{becd}}$ & $0.50 \pm 0.00^{\mathrm{g}}$ & $71.76 \pm 2.13^{\mathrm{dc}}$ & $342.19 \pm 3.07^{\mathrm{e}}$ \\
\hline 6 & Dafo & $8.11 \pm 0.70^{\mathrm{fdec}}$ & $2.65 \pm 0.04^{\mathrm{de}}$ & $15.72 \pm 0.29^{\text {ba }}$ & $4.31 \pm 0.45^{\mathrm{a}}$ & $1.12 \pm 0.03^{\mathrm{bac}}$ & $68.09 \pm 0.13^{\mathrm{f}}$ & $345.31 \pm 1.27^{\mathrm{dce}}$ \\
\hline 7 & Dinsho & $8.94 \pm 0.33^{\mathrm{a}}$ & $3.28 \pm 0.15^{\mathrm{c}}$ & $8.58 \pm 0.21^{\mathrm{h}}$ & $3.06 \pm 0.52^{\mathrm{fecd}}$ & $1.03 \pm 0.02^{\text {bdac }}$ & $75.11 \pm 0.74^{\mathrm{a}}$ & $344.03 \pm 2.30^{\text {de }}$ \\
\hline 8 & Gobe & $7.81 \pm 0.03^{\text {fgde }}$ & $2.70 \pm 0.17^{\mathrm{dce}}$ & $13.94 \pm 1.29^{\mathrm{edf}}$ & $1.99 \pm 0.46^{\mathrm{g}}$ & $0.98 \pm 0.34^{\mathrm{bdc}}$ & $72.59 \pm 1.58^{\mathrm{bc}}$ & $354.90 \pm 4.16^{\mathrm{a}}$ \\
\hline 9 & Golden & $8.26 \pm 0.23^{\text {bdec }}$ & $3.27 \pm 0.03^{\mathrm{c}}$ & $16.64 \pm 0.23^{\mathrm{a}}$ & $2.83 \pm 0.16^{\mathrm{fecd}}$ & $0.90 \pm 0.07^{\mathrm{dec}}$ & $68.09 \pm 0.24^{\mathrm{f}}$ & $347.97 \pm 1.43^{\mathrm{dce}}$ \\
\hline 10 & Harbu & $8.35 \pm 0.44^{\text {bdac }}$ & $3.14 \pm 0.04^{\mathrm{dc}}$ & $14.48 \pm 0.27^{\text {edc }}$ & $3.21 \pm 0.08^{\text {becd }}$ & $0.71 \pm 0.20^{\mathrm{feg}}$ & $70.12 \pm 0.71^{\mathrm{de}}$ & $344.75 \pm 0.38^{\mathrm{dce}}$ \\
\hline 11 & HB 1307 & $7.64 \pm 0.33^{\text {fge }}$ & $2.59 \pm 0.12^{\mathrm{fe}}$ & $16.39 \pm 0.12^{\mathrm{a}}$ & $3.77 \pm 0.53^{\mathrm{ba}}$ & $0.88 \pm 0.05^{\mathrm{fde}}$ & $68.73 \pm 0.80^{\mathrm{fe}}$ & $348.36 \pm 4.03^{\mathrm{dc}}$ \\
\hline 12 & HB 1965 & $7.63 \pm 0.17^{\mathrm{fge}}$ & $2.39 \pm 0.63^{\mathrm{fe}}$ & $13.42 \pm 0.74^{\mathrm{gf}}$ & $2.76 \pm 0.11^{\mathrm{fecd}}$ & $0.97 \pm 0.04^{\mathrm{bdc}}$ & $72.83 \pm 1.59^{\mathrm{bc}}$ & $353.72 \pm 3.32^{\text {ba }}$ \\
\hline 13 & HB 1966 & $7.52 \pm 0.45^{\mathrm{fg}}$ & $4.18 \pm 1.03^{\mathrm{b}}$ & $12.85 \pm 0.69^{g f}$ & $2.89 \pm 0.50^{\mathrm{fecd}}$ & $1.21 \pm 0.04^{\mathrm{a}}$ & $71.34 \pm 0.59^{\mathrm{dc}}$ & $347.69 \pm 4.50^{\mathrm{dc}}$ \\
\hline 14 & Robera & $8.96 \pm 0.11^{\mathrm{a}}$ & $5.81 \pm 0.16^{\mathrm{a}}$ & $13.14 \pm 0.20^{g f}$ & $2.69 \pm 0.04^{\mathrm{fe}}$ & $1.02 \pm 0.05^{\text {bdac }}$ & $68.37 \pm 0.37^{\mathrm{f}}$ & $335.24 \pm 1.04^{\mathrm{f}}$ \\
\hline 15 & Walker & $8.97 \pm 0.28^{\mathrm{a}}$ & $2.85 \pm 0.02^{\mathrm{dce}}$ & $15.53 \pm 0.25^{\text {bac }}$ & $2.51 \pm 0.07^{\mathrm{fg}}$ & $1.00 \pm 0.13^{\mathrm{bdac}}$ & $69.25 \pm 0.58^{\mathrm{fe}}$ & $347.72 \pm 0.92^{\mathrm{dc}}$ \\
\hline Mear & & 8.21 & 3.17 & 13.90 & 3.03 & 0.93 & 70.76 & 346.99 \\
\hline LSD & & $0.65^{* *}$ & $0.59 * *$ & $1.15 * *$ & $0.62 * *$ & $0.23 * *$ & $1.45^{* *}$ & $4.94 * *$ \\
\hline $\mathrm{CV}$ & & 4.76 & 11.10 & 4.94 & 12.13 & 14.86 & 1.44 & 0.85 \\
\hline
\end{tabular}

Where: In each column means followed by different letters (a, b, c, d, e, etc.) are significantly different at $\alpha<0.05$. **= strongly significant at $\mathrm{P}<0.0001$ 


\subsection{Proximate and Energy Composition of Barley Varieties' Injera}

Proximate and energy content of barley varieties' porridge are presented in Table 4. Both proximate and energy composition were strongly significant $(\mathrm{P}<0.0001)$ among barley varieties' porridge. The moisture, ash, protein, crude fat, crude fiber, carbohydrate and energy content of Injera determined with range of $8.40-10.26 \%, 1.23-3.45 \%, 8.95$ $-18.08 \%, 2.33-5.55 \%, 0.62-1.49 \%, 66.86-74.91 \%$ and 337.99 - 352.76 Kcal respectively. The maximum moisture and ash content were obtained from Walker and Robera variety with value of $10.26 \%$ and $3.45 \%$ respectively. But the least moisture and ash content was obtained from Bentu and
BH 1965 variety with value $8.86 \%$ and $1.23 \%$ respectively. The maximum and minimum protein content was determined in BH07 (18.08\%) and Dinsho (8.95\%) respectively. The maximum and minimum crude fiber content was determined in HB $1965(5.55 \%)$ and Golden Eye (2.33\%) respectively. The least crude fat and $\mathrm{CHO}$ content was found from Aquila variety, while the maximum crude and $\mathrm{CHO}$ content was determined from Dinsho variety. The least and highest energy content obtained from HB1965 and Abdane variety with the value of $337.68 \mathrm{Kcal}$ and $352.76 \mathrm{Kcal}$ respectively. The energy composition was not significant among Biftu, HB 1307, Golden Eye and Abdane varieties.

Table 4. Proximate and Energy content of barley varieties' Injera.

\begin{tabular}{|c|c|c|c|c|c|c|c|c|}
\hline \multicolumn{2}{|l|}{ SN } & \multirow{2}{*}{$\begin{array}{l}\text { Moisture (\%) } \\
8.98 \pm 0.04^{\text {ih }}\end{array}$} & \multirow{2}{*}{$\begin{array}{l}\text { Ash (\%) } \\
1.85 \pm 0.01^{\mathrm{cb}}\end{array}$} & \multirow{2}{*}{$\frac{\text { Protein (\%) }}{15.24 \pm 0.76^{\text {cebd }}}$} & \multirow{2}{*}{$\begin{array}{l}\text { Crude Fiber (\%) } \\
2.72 \pm 0.50^{\text {gef }}\end{array}$} & \multirow{2}{*}{$\frac{\text { Crude fat (\%) }}{1.39 \pm 0.03^{\mathrm{a}}}$} & \multirow{2}{*}{$\begin{array}{l}\text { CHO (\%) } \\
69.82 \pm 0.91^{\text {cfed }}\end{array}$} & \multirow{2}{*}{$\begin{array}{l}\text { Energy(Kcal) } \\
352.76 \pm 1.65^{\mathrm{a}}\end{array}$} \\
\hline 1 & Abdane & & & & & & & \\
\hline 2 & Aquila & $9.14 \pm 0.02^{\mathrm{h}}$ & $1.70 \pm 0.07^{\mathrm{cd}}$ & $15.91 \pm 0.13^{\mathrm{b}}$ & $3.40 \pm 0.10^{\mathrm{cbd}}$ & $0.62 \pm 0.13^{\mathrm{f}}$ & $67.08 \pm 0.15^{\mathrm{i}}$ & $346.16 \pm 0.80^{\mathrm{cd}}$ \\
\hline 3 & Aruso & $10.02 \pm 0.09^{\mathrm{bc}}$ & $1.66 \pm 0.05^{\mathrm{cd}}$ & $13.53 \pm 0.63^{g f}$ & $2.96 \pm 0.13^{\text {gcefd }}$ & $1.11 \pm 0.03^{\mathrm{c}}$ & $70.72 \pm 0.55^{\mathrm{cbd}}$ & $346.97 \pm 0.39^{\text {cbd }}$ \\
\hline 4 & Bentu & $8.86 \pm 0.01^{\mathrm{i}}$ & $1.58 \pm 0.00^{\mathrm{d}}$ & $14.39 \pm 0.97^{\text {gef }}$ & $3.44 \pm 0.08^{\mathrm{cb}}$ & $0.81 \pm 0.03^{\mathrm{d}}$ & $70.94 \pm 0.86^{\mathrm{cb}}$ & $348.50 \pm 0.027^{\mathrm{cb}}$ \\
\hline 5 & Biftu & $8.96 \pm 0.19^{\text {ih }}$ & $1.68 \pm 0.04^{\mathrm{cd}}$ & $13.04 \pm 0.55^{\mathrm{g}}$ & $2.93 \pm 0.12^{\text {gcefd }}$ & $1.12 \pm 0.07^{\mathrm{c}}$ & $72.27 \pm 0.56^{\mathrm{b}}$ & $351.35 \pm 1.07^{\mathrm{a}}$ \\
\hline 6 & Dafo & $9.14 \pm 0.28^{\mathrm{h}}$ & $1.52 \pm 0.04^{\mathrm{ed}}$ & $16.33 \pm 1.80^{\mathrm{cb}}$ & $3.15 \pm 0.30^{\text {cefd }}$ & $0.83 \pm 0.04^{\mathrm{d}}$ & $69.03 \pm 1.85^{\text {gfeh }}$ & $348.90 \pm 0.32^{b}$ \\
\hline 8 & Gobe & $9.95 \pm 0.15^{\mathrm{dc}}$ & $2.01 \pm 0.54^{\mathrm{b}}$ & $16.55 \pm 0.26^{\mathrm{b}}$ & $3.84 \pm 0.32^{\mathrm{b}}$ & $0.78 \pm 0.09^{\mathrm{d}}$ & $66.86 \pm 0.26^{\mathrm{i}}$ & $340.72 \pm 0.79^{\mathrm{e}}$ \\
\hline 9 & Golden & $9.39 \pm 0.11^{\mathrm{g}}$ & $1.65 \pm 0.12^{\mathrm{cd}}$ & $16.53 \pm 1.72^{\mathrm{b}}$ & $2.33 \pm 0.12^{\mathrm{g}}$ & $1.05 \pm 0.02^{\mathrm{c}}$ & $69.05 \pm 1.99^{\text {gfeh }}$ & $351.79 \pm 1.29^{\mathrm{a}}$ \\
\hline 10 & Harbu & $9.52 \pm 0.11^{\mathrm{gf}}$ & $1.62 \pm 0.08^{\mathrm{cd}}$ & $15.22 \pm 0.64^{\text {cebd }}$ & $2.62 \pm 0.06^{\mathrm{gf}}$ & $0.67 \pm 0.00^{\mathrm{ef}}$ & $70.34 \pm 0.62^{\mathrm{ced}}$ & $348.30 \pm 0.28^{\mathrm{cbd}}$ \\
\hline 11 & НВ 1307 & $8.40 \pm 0.05^{\mathrm{j}}$ & $1.25 \pm 0.00^{\mathrm{gf}}$ & $18.08 \pm 0.26^{\mathrm{a}}$ & $3.15 \pm 0.07^{\mathrm{cefd}}$ & $0.76 \pm 0.06^{\mathrm{ed}}$ & $68.35 \pm 0.40^{\text {gfih }}$ & $352.61 \pm 0.46^{\mathrm{a}}$ \\
\hline 12 & НВ 1965 & $9.76 \pm 0.07^{\mathrm{ed}}$ & $1.23 \pm 0.02^{\mathrm{g}}$ & $15.01 \pm 0.13^{\mathrm{ced}}$ & $5.55 \pm 0.88^{\mathrm{a}}$ & $0.77 \pm 0.06^{\mathrm{ed}}$ & $67.69 \pm 0.95^{\text {ih }}$ & $337.68 \pm 3.45^{\mathrm{f}}$ \\
\hline 13 & НВ 1966 & $9.64 \pm 0.08^{\text {ef }}$ & $1.55 \pm 0.02^{\mathrm{ed}}$ & $15.95 \pm 0.42^{\text {cbd }}$ & $2.77 \pm 0.20^{\text {gefd }}$ & $0.80 \pm 0.06^{\mathrm{d}}$ & $69.29 \pm 0.68^{\text {gfed }}$ & $348.15 \pm 0.59^{\mathrm{cbd}}$ \\
\hline 14 & Robera & $10.21 \pm 0.14^{\mathrm{ba}}$ & $3.45 \pm 0.04^{\mathrm{a}}$ & $14.75 \pm 0.47^{\mathrm{efd}}$ & $2.58 \pm 0.10^{\mathrm{gf}}$ & $1.28 \pm 0.11^{\mathrm{b}}$ & $67.91 \pm 0.66^{\mathrm{gih}}$ & $341.43 \pm 0.58^{\mathrm{e}}$ \\
\hline \multicolumn{2}{|c|}{ Mean } & 9.49 & 1.70 & 14.97 & 3.19 & 0.97 & 69.53 & 347.35 \\
\hline \multicolumn{2}{|c|}{ LSD } & 0.20 & 0.25 & 1.40 & $0.66 * *$ & $0.10 * *$ & $1.70 * *$ & $2.34 * *$ \\
\hline \multicolumn{2}{|c|}{$\mathrm{CV}$} & 1.27 & 8.73 & 5.58 & 12.41 & 6.43 & 1.57 & 0.40 \\
\hline
\end{tabular}

Where: In each column means followed by different letters ( $\mathrm{a}, \mathrm{b}, \mathrm{c}, \mathrm{d}$, e, etc.) are significantly different at $\alpha<0.05$. $* *=$ strongly significant at $\mathrm{P}<0.0001$

The mean proximate and energy composition determined in both barley varieties' porridge and Injera conceded with nutrient content of barley products per 100 $\mathrm{g}$ as commonly consumed in Ethiopia food 368, 9.1, 8.5, $2.0,79.0,2.2,1.4,17.0,294,6.3[23,21]$. The proximate composition of barley grain ranges $78-83,7.6-14.4,1.3$ $-2.8,4.0-8.0$ and $2.0-5.0$ for carbohydrate, protein, fat, crude fiber and ash respectively [24, 22]. Lipid concentration of barley generally ranges from 2 to $3 \%$ [25] with reports of cultivars as high as 5.3\% [26]. The ash content, gross mineral matter of barley ranges from 2.0 to $3.0 \%$ with low ash occurring in hulless types. Barley hulls contain around $6.0 \%$ ash [27]. Mineral contain in barley ranges for $\mathrm{Na}, \mathrm{K}, \mathrm{Ca}, \mathrm{Mg}, \mathrm{Fe}$ and $\mathrm{Zn}$ for raw barley 3, $270,20,65,3.0$, and $2.1 \mathrm{mg} / \mathrm{per} 100 \mathrm{~g}$ [23]. Variables which are difficult to control such as soil composition, moisture, temperature and amount of sunlight, can produce location and seasonal variation in the grain composition [28]. As the proximate and energy compositions of barley varieties porridge and Injera shown on Tables 3 and 4 indicated; Aquila, Bentu, Gobe and $\mathrm{BH} 1965$ varieties among the best for porridge in terms of energy. While, Abdane, Biftu, Golden Eye and HB 1307 varieties had superior for Injera in terms energy.

\subsection{Sensory Evaluation Data}

Sensory evaluation is defined as a scientific discipline used to evoke, measure, analyze, and interpret those responses to products that are perceived by the senses of sight, smell, touch, taste, and hearing [29]. While acceptable color of a food varies depending on cultural, geographic and sociological aspects of a given population, certain food groups are acceptable only if they fall within a certain color range [30].

\subsection{Sensory Evaluation of Porridge}

Barley varieties' sensory acceptability of porridge are shown in Table 5. The tested porridge sensory attributes among barley varieties had significant $(\mathrm{P}<0.0001)$ differences in color, texture, mouth feel, taste, odor and overall acceptability. The mean preference of porridge for 
color, texture, mouth feel, taste, odor and overall acceptability were $6.59,5.96,6.31,5.90,5.97$ and 6.47 respectively. Barley varieties porridge was accepted with least score given for Walker porridge texture with the value of 3.89(dislike moderately) and the most preferred score 7.68
(Like moderately) was given for Gobe porridge color. Bentu variety was mostly preferred for overall acceptability but not significant among Robera, HB 1966, Gobe and Aquila varieties for overall acceptability score.

Table 5. Sensory evaluation of porridge preference for selected food barley varieties.

\begin{tabular}{|c|c|c|c|c|c|c|c|}
\hline \multirow{2}{*}{ S.N } & \multirow{2}{*}{ Sample name } & \multicolumn{6}{|c|}{ Porridge Sensory Attributes } \\
\hline & & Color & Texture & Mouth feel & Taste & Odor & Overall acceptability \\
\hline 1 & Abdane & $6.38 \pm 1.74^{\text {ef }}$ & $5.48 \pm 1.81^{\text {fe }}$ & $6.58 \pm 1.22^{\text {ba }}$ & $5.63 \pm 2.00^{\text {ed }}$ & $5.86 \pm 1.84^{\mathrm{bc}}$ & $6.59 \pm 1.55^{\text {edf }}$ \\
\hline 2 & Aquila & $7.22 \pm 1.31^{\mathrm{bac}}$ & $6.79 \pm 1.42^{\text {ba }}$ & $6.78 \pm 1.33^{\text {ba }}$ & $6.30 \pm 1.58^{\mathrm{bc}}$ & $6.32 \pm 1.83^{\text {ba }}$ & $7.13 \pm 1.25^{\text {bac }}$ \\
\hline 3 & Aruso & $6.48 \pm 1.62^{\text {edf }}$ & $6.73 \pm 1.52^{\text {ba }}$ & $6.37 \pm 1.50^{\mathrm{bc}}$ & $6.54 \pm 1.56^{\text {ba }}$ & $6.32 \pm 1.81^{\mathrm{ba}}$ & $6.84 \pm 1.60^{\mathrm{ebdc}}$ \\
\hline 4 & Biftu & $7.05 \pm 1.66^{\mathrm{c}}$ & $6.30 \pm 1.71^{\mathrm{bcd}}$ & $6.79 \pm 1.44^{\text {ba }}$ & $6.11 \pm 1.80^{\mathrm{bdc}}$ & $6.05 \pm 1.68^{\mathrm{bc}}$ & $6.68 \pm 1.59^{\mathrm{edfc}}$ \\
\hline 5 & Bentu & $7.57 \pm 1.29^{\text {ba }}$ & $7.21 \pm 1.19^{\mathrm{a}}$ & $7.21 \pm 1.62^{\mathrm{a}}$ & $6.86 \pm 1.09^{\mathrm{a}}$ & $6.67 \pm 1.45^{\mathrm{a}}$ & $7.33 \pm 1.41^{\mathrm{a}}$ \\
\hline 6 & Dafo & $5.43 \pm 1.70^{\mathrm{hi}}$ & $4.98 \pm 1.76^{\mathrm{f}}$ & $6.21 \pm 1.44^{\mathrm{bc}}$ & $5.21 \pm 1.94^{\mathrm{ef}}$ & $5.16 \pm 2.13^{d}$ & $5.70 \pm 2.06^{\mathrm{h}}$ \\
\hline 8 & Gobe & $7.68 \pm 1.23^{\mathrm{a}}$ & $6.51 \pm 1.58^{\mathrm{bc}}$ & $6.79 \pm 1.27^{\text {ba }}$ & $6.54 \pm 1.78^{\mathrm{ba}}$ & $6.27 \pm 1.74^{\text {ba }}$ & $7.06 \pm 1.58^{\text {bdac }}$ \\
\hline 9 & Golden Eye & $5.84 \pm 1.82^{\mathrm{hg}}$ & $5.16 \pm 1.81^{\mathrm{f}}$ & $5.63 \pm 1.46^{\mathrm{c}}$ & $5.10 \pm 1.96^{\mathrm{f}}$ & $5.62 \pm 1.83^{\mathrm{dc}}$ & $5.62 \pm 1.92^{\mathrm{h}}$ \\
\hline 10 & Harbu & $6.49 \pm 1.42^{\text {edf }}$ & $6.06 \pm 1.45^{\mathrm{cd}}$ & $6.58 \pm 1.17^{\text {ba }}$ & $6.10 \pm 1.64^{\mathrm{bdc}}$ & $6.00 \pm 1.67^{\mathrm{bc}}$ & $6.57 \pm 1.60^{\mathrm{ef}}$ \\
\hline 11 & HB 1965 & $6.87 \pm 1.35^{\text {edc }}$ & $5.95 \pm 1.66^{\mathrm{ed}}$ & $6.37 \pm 1.34^{\mathrm{bc}}$ & $6.03 \pm 1.64^{\mathrm{dc}}$ & $6.08 \pm 1.63^{\mathrm{bc}}$ & $6.65 \pm 1.48^{\text {edfc }}$ \\
\hline 12 & НВ 1966 & $7.14 \pm 1.70^{\mathrm{bc}}$ & $6.67 \pm 1.81^{\mathrm{b}}$ & $6.53 \pm 1.31^{\text {ba }}$ & $6.35 \pm 1.80^{\mathrm{bc}}$ & $6.63 \pm 1.47^{\mathrm{a}}$ & $7.19 \pm 1.48^{\text {ba }}$ \\
\hline 13 & HB 1307 & $6.24 \pm 1.82^{\mathrm{gf}}$ & $5.11 \pm 2.04^{\mathrm{f}}$ & $6.22 \pm 1.48^{\mathrm{bc}}$ & $5.40 \pm 2.01^{\mathrm{ef}}$ & $5.84 \pm 1.76^{\mathrm{bc}}$ & $6.03 \pm 1.93^{\mathrm{gh}}$ \\
\hline 14 & Robera & $6.94 \pm 1.60^{\mathrm{dc}}$ & $6.56 \pm 1.57^{\mathrm{bc}}$ & $5.58 \pm 1.84^{\mathrm{c}}$ & $6.38 \pm 1.65^{\text {bac }}$ & $6.22 \pm 1.89^{\mathrm{ba}}$ & $6.92 \pm 1.70^{\text {ebdac }}$ \\
\hline \multirow{3}{*}{15} & Mean & 6.59 & 5.96 & 6.31 & 5.90 & 5.97 & 6.47 \\
\hline & $\operatorname{LSD}(\mathrm{p}<0.05)$ & 0.50 & 0.49 & 0.83 & 0.49 & 0.48 & 0.47 \\
\hline & $\mathrm{CV}$ & 21.50 & 23.73 & 20.60 & 23.75 & 23.46 & 21.05 \\
\hline
\end{tabular}

Where: In each column means followed by different letters (a, b, c, d, e, etc.) are significantly different at $\alpha<0.05$.

Table 6. Sensory evaluation of injera preference for selected food barley varieties

\begin{tabular}{|c|c|c|c|c|c|c|c|c|}
\hline \multirow{2}{*}{ S.N } & \multirow{2}{*}{$\begin{array}{l}\text { Sample } \\
\text { name }\end{array}$} & \multicolumn{7}{|c|}{ Injera Sensory Attributes } \\
\hline & & Gas hole distribution & Color & Texture & Mouth feel & Taste & Odor & Overall acceptability \\
\hline 1 & Abdane & $5.94 \pm 2.52^{\text {gh }}$ & $5.99 \pm 2.08^{\mathrm{e}}$ & $5.59 \pm 2.24^{\mathrm{fg}}$ & $3.82^{\mathrm{f}}$ & $5.54 \pm 1.95^{\mathrm{d}}$ & $5.49 \pm 1.94^{\mathrm{d}}$ & $6.25 \pm 2.04^{\mathrm{e}}$ \\
\hline 2 & Aquila & $7.04 \pm 1.77^{\text {bac }}$ & $6.94 \pm 1.45^{\text {ba }}$ & $6.65 \pm 1.57^{\text {ba }}$ & $5.91^{\mathrm{bc}}$ & $5.76 \pm 1.74^{\mathrm{dc}}$ & $5.72 \pm 1.83^{\mathrm{dc}}$ & $7.13 \pm 1.41^{\mathrm{a}}$ \\
\hline 3 & Aruso & $6.96 \pm 1.64^{\text {bdac }}$ & $6.68 \pm 1.46^{\mathrm{bc}}$ & $6.88 \pm 1.04^{\mathrm{a}}$ & $6.77^{\mathrm{a}}$ & $6.31 \pm 1.69^{\mathrm{a}}$ & $6.10 \pm 1.69^{\mathrm{bac}}$ & $7.03 \pm 1.52^{\text {ba }}$ \\
\hline 4 & Biftu & $6.79 \pm 1.81^{\text {ebdac }}$ & $6.76 \pm 1.49^{\text {bac }}$ & $6.53 \pm 1.68^{\text {bdac }}$ & $6.59^{\mathrm{ab}}$ & $6.09 \pm 1.77^{\text {bac }}$ & $6.22 \pm 1.69^{\text {ba }}$ & $7.15 \pm 1.51^{\mathrm{a}}$ \\
\hline 5 & Bentu & $7.15 \pm 1.83^{\mathrm{a}}$ & $7.13 \pm 1.07^{\mathrm{a}}$ & $6.57 \pm 1.34^{\mathrm{bac}}$ & $5.95^{\mathrm{bc}}$ & $6.00 \pm 1.61^{\text {bdac }}$ & $6.22 \pm 1.55^{\text {ba }}$ & $6.94 \pm 1.56^{\mathrm{ba}}$ \\
\hline 6 & Dafo & $6.44 \pm 1.67^{\text {egdf }}$ & $6.60 \pm 1.45^{\mathrm{bdc}}$ & $6.16 \pm 1.49^{\text {bdec }}$ & $5.73^{\mathrm{cd}}$ & $6.15 \pm 1.50^{\mathrm{bac}}$ & $6.15 \pm 1.88^{\text {bac }}$ & $6.88 \pm 1.62^{\text {bac }}$ \\
\hline 8 & Gobe & $6.00 \pm 2.60^{\mathrm{gfh}}$ & $6.47 \pm 1.92^{\mathrm{dc}}$ & $6.01 \pm 1.83^{\text {feg }}$ & $4.91^{\mathrm{e}}$ & $6.07 \pm 2.09^{\mathrm{bac}}$ & $6.47 \pm 1.67^{\mathrm{a}}$ & $6.79 \pm 1.99^{\text {bdac }}$ \\
\hline 9 & Golden & $7.12 \pm 1.70^{\mathrm{ba}}$ & $6.91 \pm 1.33^{\text {bac }}$ & $6.63 \pm 1.45^{\text {ba }}$ & $5.95^{\mathrm{bc}}$ & $6.25 \pm 1.66^{\mathrm{ba}}$ & $6.10 \pm 1.61^{\mathrm{bac}}$ & $6.99 \pm 1.23^{\mathrm{ba}}$ \\
\hline 10 & Harbu & $6.69 \pm 2.13^{\text {ebdac }}$ & $6.79 \pm 1.52^{\mathrm{bac}}$ & $6.10 \pm 1.77^{\mathrm{dec}}$ & $5.05^{\mathrm{de}}$ & $5.88 \pm 1.91^{\text {bdac }}$ & $6.06 \pm 1.81^{\mathrm{bac}}$ & $6.78 \pm 1.53^{\text {bdac }}$ \\
\hline 11 & HB 1965 & $4.97 \pm 1.82^{\mathrm{i}}$ & $6.19 \pm 1.02^{\mathrm{ed}}$ & $5.56 \pm 1.24^{\mathrm{g}}$ & $5.91^{\mathrm{cb}}$ & $6.34 \pm 1.38^{\mathrm{a}}$ & $6.26 \pm 0.99^{\mathrm{ba}}$ & $6.35 \pm 1.09^{\mathrm{ed}}$ \\
\hline 12 & HB 1966 & $6.59 \pm 1.65^{\text {ebdc }}$ & $6.59 \pm 1.74^{\mathrm{bdc}}$ & $6.06 \pm 1.46^{\mathrm{fde}}$ & $6.41^{\mathrm{abc}}$ & $5.97 \pm 1.70^{\text {bdac }}$ & $6.01 \pm 1.54^{\mathrm{bac}}$ & $6.71 \pm 1.70^{\text {ebdac }}$ \\
\hline 13 & НВ 1307 & $5.68 \pm 1.55^{\mathrm{h}}$ & $6.74 \pm 1.30^{\mathrm{bac}}$ & $6.06 \pm 1.58^{\mathrm{fde}}$ & $5.77^{\mathrm{cd}}$ & $5.96 \pm 1.53^{\text {bdac }}$ & $6.13 \pm 1.54^{\mathrm{bac}}$ & $6.44 \pm 1.61^{\mathrm{edc}}$ \\
\hline 14 & Robera & $6.51 \pm 1.50^{\text {edfc }}$ & $6.60 \pm 1.35^{\mathrm{bdc}}$ & $6.32 \pm 1.47^{\text {bdec }}$ & $5.82^{\mathrm{c}}$ & $5.87 \pm 1.66^{\text {bdac }}$ & $6.04 \pm 1.77^{\text {bac }}$ & $6.90 \pm 1.44^{\mathrm{bac}}$ \\
\hline Mean & & 6.47 & 6.65 & 6.25 & 5.74 & 6.00 & 6.06 & 6.78 \\
\hline $\mathrm{CV}$ & & 24.46 & 20.21 & 23.34 & 22.25 & 23.89 & 23.02 & 20.28 \\
\hline LSD & & $0.53^{* *}$ & $0.45^{* *}$ & $0.49 * *$ & $0.76 * *$ & $0.48^{* *}$ & $0.47 * *$ & $0.46^{* *}$ \\
\hline
\end{tabular}

Where: In each column means followed by different letters (a, b, c, d, e, etc.) are significantly different at $\alpha<0.05$.

Barley varieties' sensory acceptability of data of Injera are shown in Table 6. Among barley varieties had significant $(\mathrm{P}<0.0001)$ differences in gas hole distribution, color, texture, mouth feel taste and odor preferences, but not significant among Robera, HB 1966, Harbu, Golden Eye, Gobe, Dinsho, Dafo Bentu Biftu, Aruso and Aquila varieties for overall acceptability score. All varieties were preferred for tasted sensory qualities varied from the least disliked moderately (3.82) to the highest liked moderately (7.15) as shown in the Table 6. The highest score for gas whole distribution (eye) and color were 7.15 and 7.13 respectively belonging to Bentu variety and the lowest preferred were HB 1665 and Abdane variety for eye (4.97) and color (5.99) respectively. Similarly Aruso variety had preferred most for texture, mouth feel, and taste with the scores $6.88,6.77$ and 6.31 respectively. The least score found for mouth feel, taste, odor and overall acceptability were given for Abdane variety with 3.82, 5.54, 5.49 , and 6.25, while the least texture (5.56) score provided for $\mathrm{BH}$ 1665. Gobe and Biftu variety had the highest preferred for odor (6.64) and overall acceptability (7.15) 
respective. The overall acceptability was not significant different among most barley variety except the inferior liked varieties such as Abdane, HB 19665, HB 1307 and Walker varieties. among A good Injera is soft, with uniformly distributed gas holes on its top surface and nonstick top and bottom surfaces, is supple (rolls easily), and has a slightly sour taste [31 \& 32]. The appearance, size, and distribution of gas holes on the Injera surface and its taste and texture all impact the preference and acceptability of Injera.

\section{Conclusion and Recommendations}

Fifteen released food barley varieties were determined for physicochemical and sensory qualities. This study revailed that barley variety had different merits for tested physicals, chemicals and sensory qualities parameters. Accordingly, barley varieties such as Aquila, Bentu and Gobe were preferred for porridge preparation and utilization. While Biftu, HB 1307 and Golden barley varieties were better for Injera preparation and consumption. Generally, depending on the overall yield, physicals, nutrients, energy and sensory qualities Bentu, Gobe, Aquila and HB 1307 varieties recommended for the intended users. In the future, incorporation of nutritional evaluation may be necessary during regional variety verification trails.

\section{Acknowledgements}

The authors are grateful to Food Science Research Directorate Staff for their invaluable support and encouragement, and IQQO's Research centers for provided study materials. Special thanks goes to Agricultural Growth Program II for financing this study.

\section{References}

[1] Fischbeck, G. 2002. "Contribution of barley to agriculture: A brief overview”, in G. A. Slafer, J. L.

[2] Bekele, B., Makkouk, K. M., Yusuf, A., Alemayu, F., Lencho, A. 2001. Occurrence and distribution of barley yellow dwarf virus (BYDV) isolates in central Ethiopia. Inter. J. Pest. Mande. t, 47. pp 115-119.

[3] Jemal M., Semeneh S., 2016. Revisit to Ethiopian traditional barley-based food. Journal of Ethnic Foods. J Ethn Foods 3 (2016) 135e141.

[4] James Warner Tim Stehulak Leulsegged Kasa, 2013. WoredaLevel Crop Production Rankings in Ethiopia: A Pooled Data Approach. International Food Policy Research Institute (IFPRI) Addis Ababa, Ethiopia

[5] The federal democratic republic of Ethiopia. Central statistical agency agricultural sample survey 2017/18 volume I report on area and production of major crops

[6] Ministry of Agriculture. Plant variety release, protection and seed quality control directorate. Crop variety register issue No. 21. June, 2018. Addis Ababa, Ethiopia
[7] Metayer J. P., Grosjean F., Castaing J. Study of variability in French cereals. Animal Feed Science and Technology. 1993. 43(1-2). P. 87-108.

[8] Rodehutscord M., Ruckert C., Maurer H. P., Schenkel H., Schipprack W., Knudsen K. E. B., Schollenberger M., Laux M., Eklund M., Siegert W., Mosenthin R. Variation in chemical composition and physical characteristics of cereal grains from different genotypes. Archives of Animal Nutrition. 2016. 70(2). P. 87-107.

[9] Jilal, A 2011, 'Assessment of genetically diverse international barley germplasm for development of food product applications', $\mathrm{PhD}$ thesis, Southern Cross University, Lismore, NSW.

[10] Yadesa Abeshu, Esayas Abrha. Evaluation of Proximate and Mineral Composition Profile for Different Food Barley Varieties Grown in Central Highlands of Ethiopia. World Journal of Food Science and Technology. Vol. 1, No. 3, 2017, pp. 97-100.

[11] Izydorczyk MS, Storsley J, Labossiere D, MacGregor A. W, Rossnagel BG (2000) Variation in total and soluble b-glucan content in hulless barley: effects of thermal, physical, and enzymic treatments. J of Agric and Food Chem 48:982-989.

[12] Karababa Ersan (2005). Physical properties of popcorn kernels. Journal of Food Engineering. WWW.elsevier.Com/locate/jfoodeng.1-8.

[13] AOAC (Association of Official Analytical Chemists). 2000. Official methods of Analysis of AOAC International, $17^{\text {th }}$ edition, Vol. II. Washington, DC, USA

[14] AOAC Official Method 975.03. Metals in Plants and Pet Foods. Atomic Absorption Spectrophotometric Method. First Action 1975 and Final Action 1988.

[15] Bultosa, G., \& Taylor, J. R. (2004). Paste and gel properties and in vitro digestibility of tef [Eragrostis tef (Zucc.) Trotter] starch. Starch-Stärke, $56 \quad$ (1), 20-28. doi:10.1002/star.200200191

[16] Fitsum Woldemariam, Ali Mohammed, Tadesse Fikre and Hailay Gebremedhin, 2019. Optimization of amaranths-teffbarley flour blending ratios for better nutritional and sensory acceptability of Injera. Cogent Food \& Agriculture (2019), 5: 1565079. https://doi.org/10.1080/23311932.2019.1565079

[17] İlker E, Altınbaş M, Tosun M (2009) Selection for test weight and kernel weight in high yielding wheat using a safety-fi rst index. Turk J Agric For 33: 37-45.

[18] Ullrich, S. E., Clancy, J. A., Eslick, R. F., and Lance, R. C. M. (1986). Beta-glucan content and viscosity of waxy barley. J. Cereal Sci, 4: 279 - 285.

[19] Qi, J. C., Zhang, G. P., Zhou, M. X. (2006). Protein and hordein content in barley seeds as affected by nitrogen level and their relationship to beta-amylase activity. J. Cereal Sci., 43, 102-107.

[20] Arendt, E. K., Zannini, E. (2013). Cereal Grains for the Food and Beverage Industries. Woodhead Publishing, Cambridge. 485 .

[21] Izydorczyk., M. S., Storsley., J; Labossiere., D; Mac-Gregor., A. W; Rossnagel., B. G. (2000). Variation in total and soluble b-glucan content in hulless barley: Effects of thermal, physical and enzymic treatments. J. Agric. Food Chem, 48: 982-989. 
[22] Quinde-Axtell, Z., Powers, P., and Baik, B. K., (2006). Retardation of discolouration in barley flour gel and dough. Cereal Chemistry, 83: 385-390.

[23] EHNRI [Ethiopian Health and Nutrition Research Institute]. 1997. Food composition table for use in Ethiopia 1968-1997. Part III. EHNRI, Addis Ababa, Ethiopia.

[24] Consensus document on compositional considerations for new varieties of Barley (hordeum vulgare 1.): key food and feed nutrients and anti-nutrients (2004). Env/jm/mono (2004)20

[25] Welch, R. W., (1978). Genotypic variation in oil and protein in barley grain. J. Sci. Fd. Agric, 29: 953-958.

[26] Bhatty, R. S., and Rossnagel, B. G. (1980). Lipids and fatty acid composition of Riso 1508 and normal barley. Cereal Chem, 57 (6): 382-386.

[27] Kent, N. L., and Evers, A. D. (1994). Technology of Cereals, 4th ed. Elsevier Science Ltd., Oxford.
[28] Duffus, C. M., and Cochrane, M. P. (1993). "Formation of the barley grain - Morphology, physiology and biochemistry", in A. W. MacGregor and R. S. Bhatty (eds.), Barley: Chemistry and technology, AACC, St. Paul, Minnesota, USA, pp. 31 72 .

[29] Stone, H and Sidel, JL. 1993. Sensory Evaluation Practices. 2nd ed. Academic Press: San Diego.

[30] Baik, B. K., Quinde, Z., and Ullrich, S. E. (2005). Improvement of barley-based food product colour. Proceedings of the 18th Triennial North American Barley Researchers Workshop and 4th Canadian Barley Symposium.

[31] Yetneberk, S., H. L. deKock, L. W. Rooney, and J. R. N. Taylor. 2004. Effects of sorghum cultivar on Injera quality. Cereal Chem. 8: 314-321. doi: 10.1094/CCHEM.2004.81.3.314

[32] Zegeye, A. 1997. Acceptability of injera with stewed chicken. Food Qual. Prefer. 8:293-295. doi: 10.1016/S09503293(96)00055-9 